\title{
Analgesia Peridural para 0 Trabalho de Parto e para 0 Parto: Efeitos da Adição de um Opióide
}

Effects of the Association of an Opioid with Epidural Analgesia for Labor and Delivery

José Guilherme Cecatti, Rosa Inês Costa Pereira

Amaury Sanchez Oliveira, Maria José Cecarelli

\section{RESUM0}

O objetivo deste estudo foi avaliar a eficácia e segurança da associação bupivacaína com sufentanil para a analgesia no trabalho de parto e do parto por meio de um bloqueio peridural contínuo. Realizou-se um ensaio clínico duplo-cego, prospectivo e aleatório, incluindo sessenta mulheres nuliparas da Maternidade do CAISM/UNICAMP. No momento da analgesia, as mulheres foram aleatoriamente alocadas em dois grupos: $B S$, recebendo $12,5 \mathrm{mg}$ de bupivacaína com adrenalina mais $30 \mu \mathrm{g}$ de sufentanil e BP, recebendo $12,5 \mathrm{mg}$ de bupivacaina com adrenalina mais placebo. Foram avaliados os parâmetros relativos à qualidade e duração da analgesia, duração do trabalho de parto e também possiveis efeitos sobre o recém-nascido. Os resultados mostraram a superioridade da adição do sufentanil sobre o grau de analgesia durante o tempo de ação da primeira dose de anestésico local. Não houve aumento na duração do trabalho de parto depois do início da analgesia quando se compararam ambos os grupos, nem qualquer diferença quanto à via de parto. Não houve também diferenças entre os grupos com relação à avaliação dos recém-nascidos. Conclui-se que a associação de $30 \mu \mathrm{g}$ de sufentanil à primeira dose de bupivacaína é segura e eficaz, melhorando a qualidade da analgesia, sua duração e não afetando a progressão do trabalho de parto e o resultado neonatal.

PALAVRAS-CHAVE: Anestesia. Analgesia do parto. Peridural. Opióides. Resolução da gestação.

\section{Introdução}

A dor relacionada ao trabalho de parto e parto é um tema que tem preocupado cientistas, médicos, religiosos, homens e mulheres ao longo da história

Centro de Atenção Integral à Saúde da Mulher

Universidade Estadual de Campinas

Correspondência:

J. G. Cecatti

Rua Alexander Fleming, 101

13081-970 Campinas - SP, Brasil

Fax (019)788.9304

Projeto financiado pelo FAEP/UNICAMP da humanidade. A primeira vez que se empregou um método de analgesia considerado moderno em Obstetrícia foi em 1847, por Simpson, utilizando éter e clorofórmio ${ }^{2}$.

Os mecanismos envolvidos na dor associada ao trabalho de parto são bem conhecidos. Os fatores mais importantes envolvidos são: a dilatação do colo uterino, a contração e distensão das fibras miometriais, distensão do canal de parto, tração de anexos e peritônio, pressão na uretra, bexiga e outras estruturas pélvicas, além da pressão sobre as raízes nervosas do plexo lombo-sacro ${ }^{1}$.

Entretanto, a analgesia em Obstetrícia é um 
assunto sobre o qual ainda não existe consenso. Recentemente, anestesiologistas argumentaram contra preconceitos arraigados que consideram aceitável que parturientes experimentem dores intensas, mesmo sob cuidados médicos ${ }^{6}$. São relatadas ainda, posições de que métodos farmacológicos de analgesia produzem efeitos deletérios sobre a mãe e o feto, devendo ser evitados, e de que a dor seja fundamental para a adaptação do feto à vida neonatal e à integração mãe-filho, não devendo ser abolida ${ }^{2}$. A dor é um importante sinal do início do trabalho de parto e, após caracterizada a regularidade das contrações, ela pode e deve ser aliviada, já que pode afetar a contratilidade e o fluxo sangüíneo uterinos, prolongando o tempo do trabalho de parto ${ }^{19}$.

A analgesia peridural foi introduzida em Obstetrícia em $1938^{15}$. Após várias modificações técnicas para seu aperfeiçoamento, difundiu-se na América Latina, a partir dos anos cinqüenta, e ofereceu múltiplas vantagens, entre elas a inocuidade, a não-interferência na contratilidade uterina e fácil aplicação ${ }^{13}$.

Segundo Crawford ${ }^{9}$, a principal função do bloqueio peridural é o alívio da dor durante o trabalho de parto e o parto, devendo ser indicado para a maioria das parturientes. O anestésico local mais amplamente empregado na atualidade para esse fim é a bupivacaína, cujas vantagens são a duração de ação prolongada, amplo bloqueio sensitivo, bloqueio motor mínimo, pequena passagem placentária, tempo de latência curta e ausência de comprometimento fetal ${ }^{12,14}$.

A analgesia peridural proporciona um alívio da dor considerado bom por 80 a $90 \%$ das parturientes ${ }^{17}$. A administração de anestésicos locais, embora satisfatória para o controle da dor, tem sido responsabilizada por efeitos indesejáveis, como hipotensão materna secundária ao bloqueio simpático e relaxamento muscular do assoalho pélvico e da parede abdominal, levando a dificuldades na rotação interna do pólo cefálico fetal dentro do canal de parto, à abolição do reflexo de Ferguson e ao eventual prolongamento do período expulsivo, com aumento de incidência de partos instrumentais ${ }^{24}$.

Há cerca de vinte anos, a observação do efeito analgésico de opióides administrados por via espinal trouxe esperanças de se obter uma analgesia efetiva, sem bloqueio simpático ou motor, ideal para obstetrícia, pós-operatório e dores crônicas ${ }^{5}$.

Esses conhecimentos levaram à busca de um opióide que satisfizesse as necessidades obstétricas. Sabe-se que a adição de fentanil à bupivacaína peridural é capaz de produzir analgesia satisfatória durante o primeiro estágio do trabalho de parto, sem efeitos colaterais importantes para a mãe ou recém-nascido ${ }^{18}$.

O uso exclusivo de fentanil intratecal também é capaz de produzir analgesia durante o trabalho de parto, entretanto não é capaz de promover analgesia satisfatória durante o período expulsivo ${ }^{3}$, além de estar associado à alta incidência de efeitos colaterais $^{16}$.

A associação entre anestésicos locais e opióides passa a ser, então, uma excelente opção em termos de qualidade analgésica, quando administrada por via peridural. Essa combinação parece preservar os benefícios de cada droga e diminuir o risco de efeitos indesejáveis ${ }^{23}$.

Nesse sentido, a associação fentanil/ bupivacaína e, mais recentemente sufentanil/ bupivacaína, para analgesia de parto por meio de bloqueio epidural, tem sido divulgada como vantajosa por apresentar rápido início de ação, maior duração da analgesia ${ }^{20}$ e menor grau de relaxamento muscular ${ }^{8}$. Além de melhora significativa na qualidade da analgesia, observase diminuição da necessidade de anestésicos locais e menor duração do primeiro estágio do trabalho de parto. O citrato de sufentanil em animais é cerca de 625 a 4000 vezes mais potente que a da morfina e de 5 a 15 vezes mais potente que o fentanil, apresentando ainda melhor margem de segurança quanto aos efeitos cardiovasculares. No homem também é muito mais potente que a morfina, porém, a duração da analgesia é mais curta ${ }^{21}$.

Considerando a grande diversidade de esquemas para analgesia de parto propostos com o emprego do bloqueio peridural contínuo, muitos deles empregando associações de anestésicos locais com opióides, e a necessidade de familiarização com o uso do sufentanil, procedemos à elaboração de um estudo que permitisse avaliar objetivamente os efeitos da associação de anestésico local com opióide (bupivacaína e sufentanil) e de anestésico local com placebo (bupivacaína e soro fisiológico), por via peridural, para analgesia de parto em dois grupos de nulíparas, quanto à eficácia e segurança.

\section{Material e métodos}

O presente estudo é um ensaio clínico aleatório e controlado, prospectivo e duplo-cego. Após aprovação pelo Comitê de Ética e obtenção do termo de consentimento, foram selecionadas parturientes nulíparas com indicação de analgesia 
de parto, alocadas aleatoriamente em um dos dois grupos para receber por via peridural: Grupo BS (bupivacaína 12,5 $\mathrm{mg}+$ adrenalina 12,5 $\mu \mathrm{g}+$ sufentanil $30 \mu \mathrm{g}$ ) ou Grupo BP (bupivacaína $12,5 \mathrm{mg}+$ adrenalina $12,5 \mu \mathrm{g}+$ placebo $0,6 \mathrm{ml}$ soro fisiológico).

Considerando-se a variável dependente duração da analgesia e determinando o valor de $\alpha=0,05$ e $\beta=0,05$, calculou-se um tamanho de amostra de 30 pacientes para cada grupo.

Foram admitidas nesse ao estudo gestantes nulíparas em trabalho de parto atendidas no CAISM/UNICAMP que aceitaram voluntariamente participar do estudo e que seguiam os seguintes critérios de inclusão: estado físico ASA I ou II de acordo com a classificação da Sociedade Americana de Anestesiologia (ASA), nuliparidade, gestação a termo, feto único e vivo, apresentação cefálica, em trabalho de parto e com dilatação cervical igual ou inferior a $7 \mathrm{~cm}$. Foram excluídas do estudo as parturientes com diagnóstico de sofrimento fetal previamente à indicação da analgesia; aquelas que apresentassem situação obstétrica de urgência; administração prévia de opióides; alteração anatômica materna que dificultasse a punção anestésica e história de hipersensibilidade às drogas empregadas.

As gestantes admitidas neste estudo foram distribuídas aleatoriamente em um dos dois grupos, por ordem de admissão, segundo lista previamente estabelecida com o auxílio de tabela de números aleatórios. Após indicação obstétrica de analgesia de parto, as pacientes foram encaminhadas à sala de parto, onde foram registrados os dados vitais antes do procedimento anestésico.

A punção foi realizada com a paciente sentada para a injeção da primeira dose de bupivacaína, seguida da inserção do cateter peridural, e acompanhada da injeção da solução em estudo conforme o grupo, preparada por um anestesista alheio ao estudo. Após a administração da solução, a paciente foi posicionada em decúbito dorsal horizontal, com deslocamento do útero para a esquerda até a fixação do bloqueio, mudando a seguir para decúbito lateral esquerdo, sendo permitida a alternância de decúbito sempre que solicitada.

Após a fixação do bloqueio, a parturiente tinha a evolução do trabalho de parto monitorizada, com registro da contratilidade uterina e batimentos cardíacos fetais, por meio de monitor fetal e/ou clinicamente pelo médico obstetra encarregado. $\mathrm{O}$ retorno da dor durante a contração determinava a injeção suplementar de anestésico local pelo cateter na dose de $10 \mathrm{ml}$ de bupivacaina $0,125 \%$ em ambos os grupos. Foram estudadas variáveis relativas às mulheres, à gravidez, trabalho de parto e parto, intensidade da dor, complicações e resultados perinatais. A intensidade da dor foi medida pela avaliação subjetiva materna, por meio de escala visual analógica $-\mathrm{EVA}^{25}$ aos 5, 10 e 20 minutos após toda administração peridural, considerandose analgesia satisfatória valores de EVA até três. Para tanto, foram utilizadas as fichas obstétricas e anestésicas do CAISM.

Inicialmente foram analisadas as freqüências de distribuição das variáveis de controle em ambos os grupos e, em seguida, procedeu-se à análise da distribuição das variáveis dependentes. Na análise univariada, as variáveis qualitativas foram comparadas por meio do teste do $\chi^{2}$ ou exato de Fisher, as numéricas mediante teste t de Student ou do teste de Mann-Whitney ${ }^{10}$. Para as variáveis categóricas ordenadas, utilizou-se ainda o teste do $\chi^{2}$ para tendência linear.

\section{Resultados}

A análise dos resultados confirmou a homogeneidade entre os grupos para idade. A média de idade do grupo BS foi 21,37 $\pm 4,44$ anos e do Grupo BP foi 21,77 $\pm 4,15$ anos. A média ponderal para o grupo BS foi $64,55 \pm 10,49 \mathrm{~kg}$ e para o Grupo BP foi $68,70 \pm 9,87 \mathrm{~kg}$. Os grupos foram homogêneos quanto ao grau de escolaridade. Guanto ao estado físico materno, não houve diferença significativa entre os grupos. A quase totalidade dos casos teve o início espontâneo do trabalho de parto e apenas em dois o trabalho de parto foi induzido com ocitocina, sendo ambas pacientes pertencentes ao grupo BS. Cerca de $85 \%$ de todas as mulheres utilizaram ocitocina em algum momento.

O grau de dilatação cervical antes da administração da analgesia foi semelhante em ambos os grupos. A média foi $5,10 \pm 0,9 \mathrm{~cm}$ para o grupo BS e 4,70 $\pm 0,9 \mathrm{~cm}$ para o grupo BP. A Tabela 1 fornece os valores médios de todas as variáveis numéricas de controle para os dois grupos de analgesia, mostrando não haver diferença estatística entre eles em nenhum aspecto avaliado.

No momento da analgesia, as parturientes de ambos os grupos apresentavam uma intensidade de dor semelhante quando avaliada por meio de escala visual analógica durante a contração, conforme resultados expostos na Tabela 2. Em relação à via de parto, observou-se $30 \%$ de cesarianas no grupo BS contra 17\%, no grupo BP. No entanto, essa diferença não foi estatisticamente significativa. Apenas um caso do grupo BS teve parto vaginal sem o uso de fórcipe. Também não houve diferença entre os grupos quanto ao uso de fórcipe de alívio (43,3\% para o grupo BS e 56,6\% 
para o grupo BP) ou de rotação (23,3\% para o grupo BS e $26,6 \%$ para o grupo BP).

Tabela 1 - Média e desvio-padrão (DP) das variáveis de controle numéricas segundo grupo de analgesia.

\begin{tabular}{|c|c|c|c|}
\hline \multirow{3}{*}{ VARIÁVEL } & \multicolumn{2}{|c|}{ GRUPO } & \multirow{3}{*}{$\mathrm{p}$} \\
\hline & $\mathrm{BS}$ & $\mathrm{BP}$ & \\
\hline & Média DP & Média DP & \\
\hline Idade (anos) & $21,37 \pm 4,44$ & $21,77 \pm 4,15$ & $0,7203 *$ \\
\hline Peso (kg) & $64,55 \pm 10,49$ & $68,71 \pm 9,87$ & $0,1156 *$ \\
\hline Dilatação $(\mathrm{cm})$ & $5,1 \pm 0,92$ & $4,7 \pm 0,95$ & $0,1108 * *$ \\
\hline Dor (antes do bloqueio) & $7,7 \pm 1,72$ & $8,4 \pm 1,48$ & $0,1155 * *$ \\
\hline Peso RN (g) & $3.224 \pm 353$ & $3.231 \pm 435$ & $0,9467 *$ \\
\hline
\end{tabular}

* Teste t de Student.

** Teste de Mann-Whitney.

Tabela 2 - Distribuição das gestantes segundo intensidade de dor antes do bloqueio peridural e grupo de analgesia.

\begin{tabular}{crr} 
DOR ANTES (EVA) & \multicolumn{2}{c}{ GRUPO } \\
\cline { 2 - 3 } & BS & BP \\
\hline $3-6$ & 6 & 4 \\
$7-8$ & 14 & 12 \\
$9-10$ & 10 & 14 \\
TOTAL & 30 & 30 \\
\hline
\end{tabular}

$\chi_{\text {trend }}^{2}=1,15 \quad \mathrm{p}=0,283$

A Tabela 3 mostra que a distribuição das gestantes por categorias de período de latência foi significativamente diferente para os grupos. Metade das mulheres do grupo BS teve um período de latência de até 8 minutos.

Tabela 3 - Distribuição das gestantes segundo tempo de latência do bloqueio e grupo de analgesia.

\begin{tabular}{ccr} 
LATÊNCIA (min) & \multicolumn{2}{c}{ GRUPO } \\
\cline { 2 - 3 } & BS & BP \\
\hline$=8$ & 15 & 6 \\
$9-15$ & 13 & 19 \\
$>15$ & 2 & 5 \\
TOTAL & 30 & 30 \\
\hline
\end{tabular}

$\chi_{\text {trend }}^{2}=5,72 \quad \mathrm{p}=0,0167$
A qualidade da analgesia avaliada cinco minutos após a instalação do bloqueio peridural e administração da associação analgésica foi semelhante em ambos os grupos, sendo considerada adequada em quase $40 \%$ dos casos. No entanto, decorridos dez minutos, observou-se um controle da dor mais adequado no grupo que recebeu sufentanil, sendo que mais de $85 \%$ das pacientes exibiam índices satisfatórios de analgesia. Vinte minutos depois ainda se observou um melhor desempenho analgésico no grupo que recebeu opióide (Tabela 4).

Tabela 4 - Porcentagem de baixos escores de dor pela EVA após primeira dose de anestésico, segundo tempo e grupo de analgesia.

\begin{tabular}{|c|c|c|c|c|}
\hline \multirow{3}{*}{$\begin{array}{l}\mathbf{1}^{\mathrm{a}} \text { DOSE } \\
\text { TEMPO (min) }\end{array}$} & \multicolumn{2}{|c|}{ GRUPOS } & \multirow{3}{*}{$\mathrm{N}^{*}$} & \multirow{3}{*}{$\mathrm{p}^{* *}$} \\
\hline & BS & $\mathrm{BP}$ & & \\
\hline & $\%$ EVA $\leq 3$ & $\%$ EVA $\leq 3$ & & \\
\hline 5 & 39,3 & 30,0 & 58 & 0,072 \\
\hline 10 & 85,7 & 34,5 & 57 & 0,0002 \\
\hline 20 & 75,0 & 42,3 & 54 & 0,030 \\
\hline
\end{tabular}

* Algumas mulheres sem contração no momento da avaliação. $* *$ Teste de $\chi_{\text {Yates }}^{2}$

Para a segunda e terceira doses de anestésico, não houve diferença entre os grupos para os escores de dor. O grau de analgesia durante o período expulsivo pode ser considerado adequado para a maioria das parturientes, não havendo diferença significativa entre os grupos quanto à freqüência dos escores (Tabelas 5 e 6).

Tabela 5 - Porcentagem de baixos escores de dor pela EVA antes e após segunda dose de anestésico, segundo tempo e grupo de analgesia.

\begin{tabular}{|c|c|c|c|c|}
\hline \multirow{3}{*}{$\begin{array}{l}\mathbf{2}^{\mathbf{a}} \text { DOSE } \\
\text { TEMPO }(\mathrm{min})\end{array}$} & \multicolumn{2}{|c|}{ GRUPOS } & \multirow{3}{*}{$\mathrm{N}^{*}$} & \multirow{3}{*}{$\mathrm{p}^{* *}$} \\
\hline & $\mathrm{BS}$ & $\mathrm{BP}$ & & \\
\hline & $\% \mathrm{EVA} \leq 3$ & $\% \mathrm{EVA} \leq 3$ & & \\
\hline Antes & 24,0 & 10,0 & 55 & 0,273 \\
\hline 5 & 60,9 & 34,6 & 49 & 0,120 \\
\hline 10 & 86,4 & 66,7 & 49 & 0,207 \\
\hline 20 & 85,7 & 80,0 & 46 & 0,456 \\
\hline
\end{tabular}

* Algumas mulheres sem contração no momento da avaliação ou parto já ocorrido.

$* *$ Teste de $\chi_{\text {Yates }}^{2}$ ou Fischer. 
Tabela 6 - Porcentagem de baixos escores de dor pela EVA antes e após terceira dose de anestésico e no período expulsivo, segundo tempo e grupo de analgesia.

\begin{tabular}{|c|c|c|c|c|}
\hline \multirow{3}{*}{$\begin{array}{l}\mathbf{3}^{\mathbf{a}} \text { DOSE } \\
\text { TEMPO (min) }\end{array}$} & \multicolumn{2}{|c|}{ GRUPOS } & \multirow{3}{*}{$\mathrm{N}^{*}$} & \multirow{3}{*}{$\mathrm{p}^{* *}$} \\
\hline & BS & $\mathrm{BP}$ & & \\
\hline & $\% \mathrm{EVA} \leq 3$ & $\%$ EVA $\leq 3$ & & \\
\hline Antes & 31,2 & 30,0 & 36 & 1,000 \\
\hline 5 & 57,1 & 35,3 & 31 & 0,393 \\
\hline 10 & 85,7 & 62,5 & 30 & 0,154 \\
\hline 20 & 77,8 & 63,6 & 20 & 0,425 \\
\hline Expulsivo & 80,9 & 56,0 & 46 & 0,138 \\
\hline
\end{tabular}

* Algumas mulheres sem contração no momento da avaliação ou parto já ocorrido.

** Teste de $\chi_{\text {Yates }}^{2}$ ou Fischer.

O tempo de trabalho de parto decorrido entre a analgesia e a ultimação do parto vaginal, não diferiu significativamente entre os grupos. As condições de vitalidade dos RN não apresentaram diferenças entre os grupos. Houve apenas um caso e um controle com Apgar de primeiro minuto menor que 7 e nenhum aos cinco minutos. Também não houve diferença estatisticamente significativa entre os grupos quanto à necessidade e tipo de medicação suplementar administrada.

A sensação de prurido ocorreu em $36 \%$ das parturientes do grupo BS. Não houve nenhum caso de depressão respiratória e os demais efeitos colaterais ocorreram de maneira semelhante em ambos os grupos. Não houve também variação significativa entre os grupos quanto ao comportamento da pressão arterial, freqüência cardíaca, freqüência respiratória e saturação de oxigênio.

\section{Discussão}

Após a análise dos resultados, pode-se verificar marcante superioridade da associação bupivacaína/sufentanil em relação à qualidade da analgesia produzida com bupivacaína somente. Trata-se não apenas de aumento na intensidade da analgesia, mas também de prolongamento de sua duração.

A afirmação de que a analgesia peridural deva ser contra-indicada nas fases iniciais do trabalho de parto, por levar a um aumento no índice de cesarianas por distocia ${ }^{26}$, motivou discussões ${ }^{6,11} \mathrm{e}$ incentivou a realização de estudos com esse objetivo $^{7}$ que apresentaram resultados favoráveis à intervenção para alívio da dor materna assim que o trabalho de parto esteja bem estabelecido, sem prolongamento no tempo de trabalho de parto ou aumento no índice de cesarianas.

A análise das variáveis de controle confirmou a homogeneidade dos grupos de estudo, garantindo sua comparabilidade quanto à idade, peso, intensidade da dor à administração da analgesia, ao grau de dilatação cervical e peso do recémnascido. Os grupos foram ainda similares quanto à via final de parto.

A associação de sufentanil à primeira dose de anestésico local foi capaz de potencializar o efeito da bupivacaína, abreviando o tempo necessário para a obtenção de analgesia, isto é, encurtando o período de latência e reduzindo rapidamente os escores de dor pela EVA. Esse efeito foi mais marcante após a injeção da associação bupivacaína e sufentanil. Durante o período expulsivo, a distribuição de mulheres com escore de dor até 3 foi semelhante entre o dois grupos.

O tempo de permanência em analgesia de parto não diferiu entre os grupos e isso está de acordo com os estudos de Van Steenberge ${ }^{27}$, mas em discordância com os resultados de Castro et al. ${ }^{4}$ que observaram uma diminuição na duração do primeiro estágio entre as parturientes que receberam a adição de sufentanil.

Embora não tenha havido diferenças entre os dois grupos quanto ao tempo de permanência em analgesia até a ultimação do parto vaginal, os valores médios encontrados, de 2 a 3 horas, foram menores e representam cerca de 50\% do tempo relatado por outros autores ${ }^{7}$. Essas diferenças podem, não necessariamente, ser atribuídas ao procedimento anestésico. Talvez possam decorrer de diferenças de condutas obstétricas em parturientes nulíparas. Deve ser lembrado, por exemplo, que nessa instituição é rotineiro o emprego de fórcipe de alívio para a abreviação do período expulsivo em nulíparas submetidas à analgesia peridural.

A necessidade do uso de ocitocina não diferiu entre os grupos e seu emprego freqüente está em consonância com as condutas preconizadas para o controle ativo da evolução do trabalho de parto durante a analgesia peridural, que possibilitariam o alívio da dor durante a fase de máximo trabalho uterino, sem o indesejável aumento no índice de cesarianas $^{11}$.

Embora o índice de cesarianas tenha sido de $30 \%$ no grupo BS, essa freqüência não foi estatisticamente superior à observada no grupo B. Também está próxima à incidência em nossa maternidade, que apresentou um índice de 28,3\% entre as primigestas atendidas no CAISM, em 1995. Deve-se levar em conta tratar-se de um Hospital 
Universitário, com atendimento em nível terciário e, portanto, com um indice de cesarianas mais elevado que o habitual. Outros autores confirmam não haver aumento nos índices de cesarianas em decorrência da associação de opióides, mesmo com a utilização mais freqüente de analgesia peridural para o controle do trabalho de parto ${ }^{22}$.

Provavelmente um dos mais importantes aspectos do estudo, do ponto de vista de segurança de utilização, foi a não interferência do sufentanil nas condições de vitalidade fetal. Isso foi demonstrado por meio do índice de Apgar semelhante entre os dois grupos.

Outro aspecto importante desse estudo refere-se ao momento da realização da analgesia durante o trabalho de parto. É provável que a duração do tempo entre a instalação da analgesia e o momento do parto não tenha diferido significativamente entre os dois grupos. Esse tempo, em ambos os grupos, foi mais prolongado do que referem outros autores que estudaram o assunto $^{4}$, provavelmente porque as pacientes do presente estudo foram mais precocemente submetidas ao procedimento. Isso é particularmente importante em termos de condução obstétrica de nulíparas que poderiam ter as dores decorrentes da fase ativa do trabalho de parto adequadamente controladas pela analgesia, podendo, assim, ser evitado o estresse e as distocias daí decorrentes. A indicação da analgesia peridural deveria idealmente priorizar o controle da dor que está sendo suportada com dificuldade pela nulípara em trabalho de parto, em vez de ser indicada com base na dilatação cervical, como freqüentemente acontece em muitos serviços do país. Os resultados obtidos também demonstraram que esse tipo de procedimento "precocemente" realizado não esteve associado a qualquer comprometimento das condições de saúde tanto da mãe quanto do feto e recém-nascido, podendo ser utilizado com segurança na prática obstétrica e anestesiológica.

Os resultados desse estudo demonstram que, efetivamente, o sufentanil adicionado à bupivacaína na analgesia peridural em nulíparas, durante o trabalho de parto, é um procedimento mais eficaz em termos de qualidade de analgesia, isento de efeitos colaterais importantes que possam ser imputados em adição. Essa técnica, portanto, demonstrou eficácia quanto aos objetivos do estudo, o que leva a recomendá-la como uma boa alternativa para a prática obstétrica e anestesiológica, merecendo, entretanto, ainda ser avaliada mais exaustivamente com relação à sua indicação em mulheres com outras características, incluindo diferentes paridades, presença de doenças maternas concomitantes ou próprias da gravidez e alterações crônicas da vitalidade fetal.

\section{SUMMARY}

The purpose of the present study was to evaluate the efficacy and safety of the association bupivacaine with sufentanil for labor and delivery analgesia through a continuous epidural blockade, for both mother and the neonate. A randomized double blind prospective clinical trial was performed including sixty nulliparous women at the Maternity of CAISM/ UNICAMP. When requesting analgesia, the women were randomly allocated to two groups: $B S$, receiving $12.5 \mathrm{mg}$ of bupivacaine with adrenaline plus $30 \mu \mathrm{g}$ of sufentanil and $\mathrm{BP}$, receiving $12.5 \mathrm{mg}$ of bupivacaine with adrenaline plus placebo. The parameters concerning the quality and duration of analgesia, duration of labor, and also possible effects on the neonate were evaluated. The results showed the superiority of the addition of sufentanil regarding the degree of analgesia during the time of action of the first dose of the local anesthetic. There was no increase in the duration of labor after the onset of analgesia when comparing both groups, nor any difference in the route of delivery. Concerning neonate evaluation, there were no differences between the two groups. It is concluded that the association of $30 \mu \mathrm{g}$ of sufentanil with the first dose of bupivacaine is safe and efficacious. It improved the quality of analgesia, increased its duration, and did not affect the progress of labor and neonatal outcome.

KEY WORDS: Labor and delivery analgesia. Epidural. Opioid.

\section{Referências}

1. Bonica JJ. Peripherical mechanisms and pathways of parturition Pain. Br J Anaesth 1979; 51: 3S-9S.

2. Bonica JJ, McDonald JS. The pain of childbirth. In: Bonica JJ. editors. The Management of Pain, $2^{\text {nd }}$ ed. Philadelphia: Lea \& Febiger, 1990. p. 1313-43.

3. Carrie LES \& O'Sullivan GM, Seegobin R. Forum: Epidural fentanyl in labour. Anaesthesia 1981; 36: 965-9.

4. Castro LFL, Sanchez CA, Oliveira AS. Continuous epidural bupivacaine plus sufentanil for labor pain relief. Rev Chil Anestesiol 1995; 24(1S): 141.

5. Chaney MA. Side effects of intrathecal and epidural opioids. Can J Anaesth 1995; 42: 891-903.

6. Chestnut DH. Epidural anesthesia and instrumental vaginal delivery. Editorial. Anesthesiology 1991; 74 : 805-8.

7. Chestnut DH, McGrath JM, Vincent RD Jr, Penning DH, Choi WW, Bates JN, et al. Does early administration of epidural analgesia affect obstetric outcome in nulliparous women who are in spontaneous labor? Anesthesiology 1994; 80: 1201-8. 
8. Cohen SE, Tan S, Albright GA, Halpern J. Epidural fentanyl/bupivacaine mixtures for obstetric analgesia. Anesthesiology 1987; 67: 403-7.

9. Crawford JS. Lumbar epidural block in labour: a clinical analysis. Br J Anaesth 1972; 44: 66-74.

10.Dexter F. Analysis of statistical tests to compare doses of analgesics among groups. Anesthesiology 1994; 81: 610-5.

11.Dewan DM \& Cohen SE. Epidural analgesia and the incidence of cesarean section: time for a closer look. Anesthesiology 1994; 80: 1189-92.

12.Eugênio AGB, Oliveira AS, Bozza IZ, Martins JE. Bupivacaína em bloqueio peridural contínuo para analgesia obstétrica. Rev Bras Anestesiol 1974; 24: 101-11.

13.Faúndes A, Gormaz G, Cabrera L. Experiencia con analgesia epidural lumbar contínua en el parto. Bol Soc Chil Obst Ginec 1959; 24: 188-91.

14.Glosten B. Pain relief for the laboring patient: current techniques and complications. 45 $\frac{\text { th }}{\text { ASA Annual }}$ Refresher Course Lectures; 1994. 271, p. 1-7.

15.Graffagnino P, Seyler LW. Epidural Anesthesia in Obstetrics. Am J Obstet Gynecol 1938; 35: 597-602.

16.Grieco WM, Norris MC, Leighton BL, Arkoosh VA, Huffnagle HJ, Honet JE, et al. Intrathecal sufentanil labor analgesia: the effects of adding morphine or epinephrine. Anesthesiology 1993; 77: $1149-54$.

17.Hood DE, Dewan DM. Anestesia obstetrica. In: Brown D, editor. Riesgos y resultados en anestesia. Barcelona: Ediciones Doyma, 1991. p. 323-75.

18.Justins DM, Francis D, Houlton PG, Reynolds F. A controlled trial of extradural fentanil in labour. Br J Anaesth 1982; 54: 409-14.
19.Lederman RP, Lederman E, Work BA Jr, McCann DS. The relationship of maternal anxiety, plasma catecholamines and plasma cortisol to progress in labor. Am J Obstet Gynecol 1978; 132: 495-500.

20.Lirzin JD, Jacquinot P, Jorrot JC, Dailland P, Conseiller C. Effect of diluting fentanyl on epidural bupivacaine during labor analgesia. Reg Anesth 1989; 14: 279-81.

21.Monk JP, Beresford R, Ward A. Sufentanil. A review of its pharmacological properties and therapeutic use. Drugs 1988; 36: 286-313.

22.Newman LM, Perez EC, Krolick TJ, Ivankovich AD. Labor analgesia, cesarean anesthesia, and cesarean delivery rates for 18000 deliveries from 1988 through 1994. Anesthesiology 1995; 83 (3A): A968.

23.Phillips G. Continuous infusion epidural analgesia in labor: the effect of adding sufentanil to $0.125 \%$ bupivacaine. Anesth Analg 1988; 67: 462-65.

24.Sanchez-Pereles MC, Uribarri FJ, Gragera I. Bupivacaína al $0.0625 \%$ comparada con bupivacaína al $0.125 \%$ en perfusión continua por via epidutal en parto vaginal. $0.0625 \%$ bupivacaine compared with $0.125 \%$ bupivacaine continuously perfused epidurally during delivery. Rev Esp Anestesiol Reanim 1993; 40: 9-11.

25.Steinberg RB, Powell G, Hu XH, Dunn SM. Epidural sufentanil for analgesia for labor and delivery. Reg Anesth 1989; 14: 225-8.

26.Thorp JA, Eckert LO, Ang MS, Johnston DA, Peaceman AM, Parisi VM. Epidural analgesia and cesarean section for dystocia: risk factors in nulliparas. Am J Perinatol 1991; 8: 402-10.

27.Van Steenberge A, Debroux HC, Noorduin H. Extradural bupivacaine with sufentanil for vaginal delivery. Br J Anaesth 1987; 59: 1518-22. 